\title{
Retrospective analysis of 23 Chinese children with congenital hyperinsulinism undergoing pancreatectomy
}

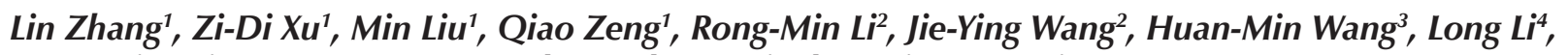 \\ Hong Qin ${ }^{3}$, Jie Yan ${ }^{1}$, Yu-Jun Wu ${ }^{1}$, Cheng Zhu ${ }^{1}$, Gui-Chen Ni ${ }^{1}$, Yan-Mei Sang ${ }^{1}$ \\ ${ }^{1}$ Department of Endocrinology, Genetics and Metabolism Centre, Beijing Children's Hospital, Capital Medical University, National \\ Centre for Children's Health, Beijing, China \\ ${ }^{2}$ Baoding Key Laboratory of Clinical Research on Children's Respiratory and Digestive Diseases/Baoding Children's Hospital, \\ Baoding, Hebei, China \\ ${ }^{3}$ Department of Surgical Oncology, Beijing Children's Hospital, Capital Medical University, National Centre for Children's Health, \\ Beijing, China \\ ${ }^{4}$ Department of Paediatric Surgery, Children's Hospital, Capital Institute of Paediatrics, Beijing, China
}

\begin{abstract}
Introduction: The aim of the study was to discuss therapeutic effect and prognosis of pancreatectomy in the treatment of congenital hyperinsulinism $(\mathrm{CHI})$.

Material and methods: A total of 23 Chinese children with $\mathrm{CHI}$, who had undergone pancreatectomy, were selected as the study objects. The clinical data, the results of the ${ }^{18}$ Fluoro-L-3-4 dihydroxyphenylalanine positron emission tomography/computerized tomography $\left({ }^{18} \mathrm{~F}-\mathrm{DOPA}\right.$ PET/CT $)$ scanning, and the diagnosis, treatment, and follow-up were analysed retrospectively.

Results: Among the 23 cases, 14 patients were diagnosed with focal-type $\mathrm{CHI}$ via a ${ }^{18} \mathrm{~F}-\mathrm{DOPA}$ PET/CT scan prior to the operation, with the lesions removed via partial pancreatectomy. After the operation, ten patients $(71 \%)$ had normal blood glucose levels, while frequent feeding was required in four patients $(29 \%)$ to control the hypoglycaemia. Three cases were diagnosed as diffuse-type CHI via preoperative scanning, two of which were treated by subtotal pancreatectomy. The other case was treated by near-total pancreatectomy, and the blood glucose level was normal following the operation. The remaining six cases were not diagnosed via the pancreatic scanning prior to the operation due to the limitation of certain conditions. Here, pancreatectomy was performed directly due to severe hypoglycaemia. Conclusions: ${ }^{18} \mathrm{~F}-\mathrm{DOPA}$ PET/CT scanning was a reliable method for determining the histological type and localizing the lesion before the operation. Partial pancreatectomy for focal-type CHI had a high cure rate. (Endokrynol Pol 2021; 72 (5): 584-585)
\end{abstract}

Key words: congenital hyperinsulinism; hypoglycaemia; pancreatectomy; ${ }^{18} \mathrm{~F}-\mathrm{DOPA} P E T / C T$ scan

\section{Introduction}

Congenital hyperinsulinism (CHI) is a genetically heterogeneous disease characterized by inappropriate insulin secretion under hypoglycaemia [1]. In this study, the therapeutic effect of pancreatectomy in the treatment of CHI was explored to provide a theoretical basis for the establishment of a clinical treatment scheme for children with $\mathrm{CHI}$.

\section{Material and methods}

A total of 23 children who had undergone pancreatectomy in Beijing Children's Hospital between April 2002 and March 2020 were selected as the study objects. All the patients met the diagnostic criteria of $\mathrm{CHI}$ [2]. The general clinical data of the 23 cases were recorded by reviewing the case data and the follow-up data, and these data were retrospectively analysed. The present retrospective study was approved by the hospital's ethics committee, and informed consent forms were signed by the parents. Following the diagnosis of CHI, 22 cases were experimentally treated with diazoxide. The therapeutic effect of the diazoxide was evaluated at least five days after the treatment. If the maximum recommended dose of diazoxide $(15 \mathrm{mg} / \mathrm{kg} / \mathrm{d})$ did not result in the standard being met after five days, the treatment was regarded as ineffective [4]. One case (case 11) was not treated with diazoxide due to the limitation of a specific therapeutic condition, with the blood glucose level maintained via medical treatment such as frequent feeding.

All 23 patients underwent at least one of the following imaging examinations: pancreatic ultrasound, computed tomography (CT), or magnetic resonance imaging (MRI). ${ }^{18} \mathrm{~F}$-DOPA PET/CT scanning was performed in 17 patients prior to the operation. The remaining six cases were not scanned for their histological type due to specific limiting conditions.

The pharmacotherapy treatment involving the diazoxide was ineffective for 22 of the 23 patients, and further surgical treatment was thus carried out. One case (case 11) underwent near-total pancreatectomy. Among the 23 cases, 11 patients underwent laparoscopic surgery and 12 received traditional open surgery.

All 23 patients underwent pancreatic biopsy and a pathological examination was performed.

The follow-up time ranged from 21 months to 17 years. During the long-term follow-up, the cure criteria were that the patient did not require frequent feeding or medication, and that the blood glucose level could be maintained within a normal range without the occurrence of diabetes mellitus. 


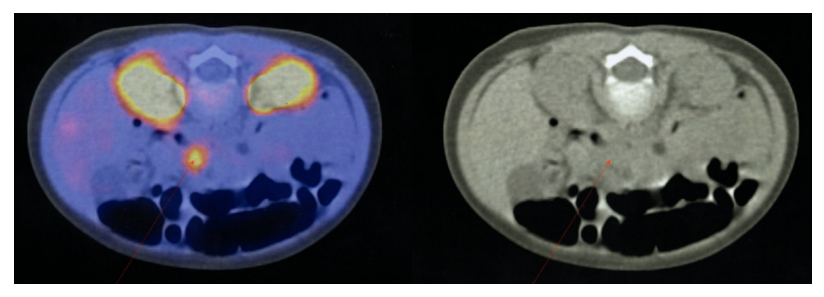

Figure 1. Image of ${ }^{18} \mathrm{~F}-\mathrm{DOPA}$ PET/CT scan in focal type CHI. Figure A: ${ }^{18} \mathrm{~F}$-DOPA PET/CT scan of case 14 indicated the location of the lesion in the pancreatic head (red arrow). Figure B: Lack of lesion in the corresponding site by CT scan

\section{Results}

\section{Focal-type congenital hyperinsulinism}

Fourteen patients were diagnosed with focal-type $\mathrm{CHI}$ by ${ }^{18} \mathrm{~F}-\mathrm{DOPA}$ PET/CT scanning, all of whom subsequently underwent partial pancreatectomy. The distribution of the pancreatic lesion is shown in Figure 1.

Ten patients were cured, and four required frequent feeding to control the hypoglycaemia. During the long-term follow-up assessment, patient 6 and patient 21 were found to be experiencing convulsions after the operation and were diagnosed with chronic epilepsy. These patients were subsequently treated with an anti-epilepsy therapy involving a combination of levetiracetam, lamotrigine, and oxcarbazepine.

\section{Diffuse-type congenital hyperinsulinism}

Three patients were diagnosed as diffuse-type $\mathrm{CHI}$ by ${ }^{18} \mathrm{~F}-\mathrm{DOPA}$ PET/CT scanning. Two of the patients underwent subtotal pancreatectomy, and one underwent near-total pancreatectomy, while all three were treated via laparoscopy.

The long-term follow-up assessment indicated that the blood glucose level had returned to normal in two of three patients. One patient (case 22) experienced frequent hypoglycaemia following the subtotal pancreatectomy and required a subcutaneous injection of octreotide $(5 \mu \mathrm{g} / \mathrm{kg} / \mathrm{d})$ to maintain the stability of the blood glucose level.

\section{Congenital hyperinsulinism with unknown preoperative histological type}

Six patients were treated via near-total pancreatectomy or subtotal pancreatectomy. Case 1 and 23 were treated via subtotal pancreatectomy, while cases $3,4,5$, and 11 were treated via near-total pancreatectomy. Case 5 was treated via laparoscopic surgery, while the remaining five were treated via traditional open surgery. The postoperative pathological examination indicated that cases $1,3,11$, and 23 were the diffuse type, while cases 4 and 5 were the focal type.

Case 1 had a normal blood glucose level at discharge, but this was found to have changed during the long-term follow-up. For case 23, oral glucose was frequently used to prevent hypoglycaemia. Among the four cases involving via near-total pancreatectomy, the patients developed diabetes at six years, one month, three years, and ten years after the operation, respectively. Case 3 controlled blood glucose level by diet control and exercise, oral hypoglycaemic drugs for case 11, and insulin for cases 4 and 5 .

\section{Discussion}

According to the histological characteristics of the pancreas, CHI can be divided into three types: focal type, diffuse type, and atypical type [5]. Children with severe hypoglycaemia and for whom medical treatment proves to be ineffective generally require further pancreatectomy. Here, 18F-DOPA PET/CT scanning should be performed as early as possible prior to the operation to determine the histological type of the pancreas and to identify the location of the lesions. In terms of focal-type $\mathrm{CHI}$, partial pancreatectomy has a high cure rate, while in terms of the diffuse type, the surgical methods are likely to be more complex, and there are likely to be significant differences in the therapeutic effects and the prognosis. Here, any patients experiencing postoperative hypoglycaemia should be treated via medications, while following the diagnosis of diabetes, individualized comprehensive treatments should be administered.

\section{Funding}

Capital Characteristic Clinic Project (Z141107002514142); Baoding Self-financing Project of Science and Technology Plan (1951ZF076)

\section{Conflict of interests}

The authors declare that they have no competing interests.

\section{Acknowledgements}

We would like to acknowledge the hard and dedicated work of all the staff that implemented the intervention and evaluation components of the study.

\section{References}

1. Yorifuji T, Horikawa R, Hasegawa T, et al. (on behalf of The Japanese Society for Pediatric Endocrinology and The Japanese Society of Pediatric Surgeons). Clinical practice guidelines for congenital hyperinsulinism. Clin Pediatr Endocrinol. 2017; 26(3): 127-152, doi: 10.1297/cpe.26.127, indexed in Pubmed: 28804205.

2. Ferrara C, Patel P, Becker S, et al. Biomarkers of Insulin for the Diagnosis of Hyperinsulinemic Hypoglycemia in Infants and Children. J Pediatr. 2016; 168: 212-219, doi: 10.1016/j.jpeds.2015.09.045, indexed in Pubmed: 26490124

3. Lord K, De León DD. Monogenic hyperinsulinemic hypoglycemia: current insights into the pathogenesis and management. Int J Pediatr Endocrinol. 2013; 2013(1): 3, doi: 10.1186/1687-9856-2013-3, indexed in Pubmed: 23384201.

4. Galcheva S, Al-Khawaga S, Hussain K. Diagnosis and management of hyperinsulinaemic hypoglycaemia. Best Pract Res Clin Endocrinol Metab. 2018; 32(4): 551-573, doi: 10.1016/j.beem.2018.05.014, indexed in Pubmed: 30086874 . 